Article

\title{
Energy and Nutrient Intake of Americans according to Meeting Current Dairy Recommendations
}

\author{
Julie M. Hess ${ }^{1, * \mathbb{C}}$, Christopher J. Cifelli ${ }^{1}(\mathbb{C})$ and Victor L. Fulgoni III ${ }^{2} \mathbb{C}$ \\ 1 National Dairy Council, Rosemont, IL 60018, USA; Chris.Cifelli@dairy.org \\ 2 Nutrition Impact LLC, Battle Creek, MI 49014, USA; vic3rd@aol.com \\ * Correspondence: Julie.hess@dairy.org; Tel.: +1-224-422-6153
}

Received: 31 August 2020; Accepted: 24 September 2020; Published: 30 September 2020

check for updates

\begin{abstract}
Most Americans do not meet dairy food recommendations from the 2015 Dietary Guidelines for Americans (DGA). This study assesses differences in nutrient intake between Americans who meet recommendations for dairy intake and those who do not, using data from the National Health and Nutrition Examination Survey from 2013-2014 and 2015-2016 ( $n=5670$ children ages 2-18 years and $n=10,112$ adults ages 19+). Among children and adults, those meeting dairy food recommendations were significantly more likely to have adequate intake (\% above Estimated Average Requirement (EAR)) of calcium, magnesium, phosphorus, riboflavin, vitamin A, vitamin B12, and zinc and consume above the Adequate Intake (AI) for potassium and choline than Americans not meeting dairy recommendations, regardless of age, sex, or race/ethnicity. Americans meeting dairy recommendations were also more likely to exceed recommendations for sodium and saturated fat but consume less added sugars. Nearly $60 \%$ of Americans 2 years and older not meeting dairy recommendations consumed calcium and magnesium below the EAR. Only about $20 \%$ of Americans who did not meet dairy recommendations consumed above the AI for potassium. Dairy foods make important and unique contributions to dietary patterns, and it can be difficult to meet nutrient needs without consuming recommended amounts of dairy foods.
\end{abstract}

Keywords: Dietary Guidelines for Americans; dietary guidance; dairy; dairy foods; nutrient adequacy

\section{Introduction}

The 2015-2020 Dietary Guidelines for Americans (DGA) recommends that American children and adults consume dairy foods daily: 2 cup-equivalent servings of low-fat or fat-free milk, cheese, yogurt, or fortified soy beverage for children 2-3 years, 2.5 servings for children $4-8$ years, and 3 servings for adolescents 9 to 18 years and all adults as part of the Healthy U.S.-Style and Healthy Vegetarian Eating Patterns [1]. The DGA bases these recommendations on the nutrient density of foods in the dairy group and their contributions to healthy eating patterns that remain within calorie, saturated fat, and sodium limits. The 2015 DGA identified the dairy group for its key contributions of calcium, phosphorus, vitamin A, vitamin D, riboflavin, vitamin B12, protein, potassium, zinc, choline, magnesium, and selenium in the diet [1]. According to food pattern modeling studies, the amounts of certain nutrients-calcium, magnesium, iron, vitamin A, riboflavin, potassium, vitamin D, and choline-in healthy eating patterns drop when dairy foods are removed from the diet. Calcium intake decreases by 68 to 88 percent, and vitamin D intake decreases by 20 to 30 percent across all age and sex groups without dairy in the diet [2].

Most Americans do not meet recommendations for dairy intake [1]. Data from the National Health and Nutrition Examination Survey (NHANES) from 2015-2016 indicate that 88 percent of the U.S. population consumes too few servings of dairy foods [3]. The only age group generally meeting dairy group recommendations is children 2-3 years, and consumption tends to fall with age [3]. 
Adults ages 20 years and older consume, on average, just 1.5 cup-equivalents of dairy foods per day [3]. While there are many possible reasons that Americans may not meet recommendations for dairy foods, including education, food security, and perceptions of dairy's impact on health, milk allergy, and lactose intolerance may also be reasons for underconsuming dairy foods. Most common among young children, milk allergy causes an immune response to milk proteins that can lead to several different symptoms and requires avoiding dairy foods $[4,5]$. In the case of milk allergy, fortified soy beverage is an appropriate choice from the DGA's "dairy group" to meet recommendations. Lactose intolerance occurs when individuals do not produce enough lactase and can cause discomfort such as bloating and diarrhea after consuming some lactose-containing dairy foods [6]. Based on self-report measures in a national sample of European American, African American, and Hispanic American adults, lactose intolerance affects approximately about $12 \%$ of Americans [7]. Lactose intolerance can be managed for most people by selecting lactose-free or reduced-lactose dairy foods [8].

Consuming less than the recommended servings of dairy foods can lead to inadequate intake of certain nutrients. The 2015 DGA lists the following ten nutrients as "shortfall nutrients" for the U.S. population: vitamin A, vitamin D, vitamin E, vitamin C, calcium, iron (for certain age/sex groups), magnesium, choline, potassium, and fiber. It further designates calcium, potassium, vitamin $D$, and fiber as "nutrients of public health concern" for the U.S. population, because their low intake "may pose a substantial public health concern" [1]. Other nutrients, including saturated fats, added sugars, and sodium are also described as nutrients of concern for Americans due to consumption rates higher than recommendations [1]. Dairy foods are an important source of several shortfall nutrients, including 3 of the 4 nutrients of public health concern, calcium, potassium, and vitamin D, indicating the importance of these foods to the nutrient density of the American diet. Milk was the top food source of calcium, potassium, and vitamin D among children 2-18 years and adults 19 and older in NHANES 2011-2014 [9] and NHANES 2003-2006 [10], respectively. Depending on products selected, however, dairy foods can also be sources of saturated fats, added sugars, and sodium.

Previous modeling work has indicated that increasing dairy food consumption to meet DGA recommendations could improve population-wide adequacy of some vitamins and minerals without increasing intake of saturated fats, added sugars, or sodium [11]. The aim of this study was to evaluate current dairy food group intakes of the U.S. population and the nutrient adequacy of those Americans who met recommendations for dairy foods compared to those Americans who did not meet dairy recommendations by using NHANES data from 2013-2016. These data were also evaluated by age, sex, and ethnicity. Our hypothesis is that Americans who meet recommendations for dairy food intake are more likely to meet nutrient needs than those who do not meet recommendations.

\section{Materials and Methods}

\subsection{Data Source and Study Population}

This analysis used data from day 1 of NHANES 2013-2014 and 2015-2016 ( $n=15,782$ after exclusions, including $n=5670$ children $2-18$ years and $n=10,112$ adults $19+$ years), which is a nationally representative sample of all noninstitutionalized Americans with over sampling of certain demographic groups. Data from pregnant and lactating females as well as participants with incomplete data, as determined by USDA, were excluded from analyses. Separate analyses were conducted for children 2-18 years, adults 19+ years, and all Americans 2+ years of age. Analyses were conducted for the overall population, for males/females separately, and for race/ethnic groups that were sampled in NHANES to provide nationally representative results: all, Hispanic, non-Hispanic Black, non-Hispanic White, and non-Hispanic Asian. Recommended servings of dairy foods were taken from the 2015-2020 DGA (Table 1). 
Table 1. Dairy intake recommendations from the 2015-2020 Dietary Guidelines for Americans.

\begin{tabular}{cc}
\hline Age Range & Recommended Dairy Intake (in Cup-Equivalents) \\
\hline $2-3$ years & 2 \\
$4-8$ years & 2.5 \\
9 year and older & 3 \\
\hline
\end{tabular}

\subsection{Dietary Intake Assessment/Data}

Intakes of energy and nutrients were from the NHANES total nutrient intake files which used the Food and Nutrient Database for Dietary Studies (FNDDS) for 2013-2014 and 2015-2016 to assess intakes [12,13]. The respective Food Patterns Equivalents Database (FPED) was used for assessing total dairy intake $[14,15]$.

Usual intakes for total dairy and nutrients were determined using the National Cancer Institute (NCI) method [16]. The NCI macros (Mixtran and Distrib) were used to generate parameter effects after covariate adjustments (day of the week of the $24 \mathrm{~h}$ recall, coded as weekend (Friday-Sunday) or weekday (Monday-Thursday) and sequence of dietary recalls (first or second)) and to estimate the distribution of usual intake. Variance estimates were obtained using the two days of intake with one-day sampling weights. The one-part NCI model was used for total dairy and nutrients since these substances are consumed on most days by most subjects.

Population groups meeting dairy recommendations and population groups not meeting dairy recommendations were established with the first day of dietary recall (in-person interview). Usual intakes and percent population below the Estimated Average Requirement (EAR) or above Adequate Intakes (AI) were calculated and compared between population groups meeting dairy recommendations and population groups not meeting dairy recommendations. The nutrients assessed in this study included the 12 nutrients that the 2015 DGA identifies as key contributions from the dairy group (calcium, phosphorus, vitamin A, vitamin D, riboflavin, vitamin B12, protein, potassium, zinc, choline, magnesium, and selenium) as well as added sugars, sodium, and saturated fat [1]. For added sugars and saturated fat, the recommended intake was set to $10 \%$ of calories of saturated fat and 10\% of calories of added sugars, in line with recommendations in the 2015 DGA [1].

\subsection{Other Variables}

Other demographic factors assessed included poverty income ratio (PIR; PIR $<1.35 ; 1.35 \leq$ PIR $\leq 1.85$; PIR $>1.85$ ), physical activity level (sedentary, moderate, vigorous), overweight or obesity, energy intake (kcal), grams of food consumed, and BMI or BMI z-score. Physical activity was divided into 3 groups based on the number of days in which vigorous exercise was performed, using the Physical Activity Questionnaire responses: sedentary, 0-3 days per week; moderate, 4-6 days per week; and vigorous, 7 days per week.

\subsection{Statistical Analyses}

All analyses were adjusted for the complex sample design of NHANES using appropriate survey weights, strata, and primary sampling units. All statistical analyses were performed with SAS software (version 9.4; SAS Institute Inc., Cary, NC, USA). Differences in demographics of population groups meeting dairy recommendations and population groups not meeting dairy recommendations were assessed using $t$-tests. Differences in the percentage of the population meeting dietary recommendations for those meeting dairy recommendations and those not meeting dairy recommendations were assessed using z-scores. Significance was set at $p<0.05$. 


\section{Results}

Most children and adults did not meet dairy food intake recommendations in NHANES 2013-2016. Approximately one-fourth $(27.8 \pm 0.11 \%)$ of children ages $2-18$ and just $13.6 \pm 0.5 \%$ of adults age 19 and older consumed the recommended number of daily dairy servings.

Children 2-18 years who met dairy food recommendations tended to be younger, male, and non-Hispanic White with "vigorous" activity levels and were less likely to be below a poverty-income ratio (PIR) of 1.35, have "moderate" activity level, be non-Hispanic black, or overweight or obese (Table 2). These children also consumed more energy (kcal) and food ( $\mathrm{g}$ ) but had lower body mass index (BMI) z-scores (Table 3).

Table 2. Demographic characteristics of U.S. subpopulations consuming above recommended daily servings of dairy foods and those consuming fewer than the recommended daily servings of dairy foods in the National Health and Nutrition Examination Survey 2013-2016 ${ }^{1}$.

\begin{tabular}{|c|c|c|c|c|c|c|}
\hline \multirow[b]{2}{*}{ Variables } & \multicolumn{3}{|c|}{ Children 2-18 Years } & \multicolumn{3}{|c|}{ Adults 19+ Years } \\
\hline & $\begin{array}{c}\text { Below RS }{ }^{2} \\
(n=4212)\end{array}$ & $\begin{array}{c}\text { Above RS }{ }^{2} \\
(n=1458)\end{array}$ & $p$-Value ${ }^{3}$ & $\begin{array}{l}\text { Below RS } \\
(n=8934)\end{array}$ & $\begin{array}{l}\text { Above RS } \\
(n=1178)\end{array}$ & $p$-Value \\
\hline Age (Years) & $10.7 \pm 0.1$ & $8.9 \pm 0.2$ & $<0.0001$ & $48.3 \pm 0.4$ & $44.0 \pm 0.8$ & $<0.0001$ \\
\hline Gender: Male (\%) & $47.0 \pm 1.0$ & $60.5 \pm 1.5$ & $<0.0001$ & $46.6 \pm 0.6$ & $67.5 \pm 1.7$ & $<0.0001$ \\
\hline \multicolumn{7}{|c|}{ Race/Ethnicity } \\
\hline Hispanic (\%) & $24.5 \pm 3.1$ & $22.9 \pm 3.0$ & 0.2862 & $15.1 \pm 1.7$ & $14.8 \pm 2.1$ & 0.7930 \\
\hline Non-Hispanic White (\%) & $50.2 \pm 4.0$ & $56.2 \pm 4.0$ & 0.0026 & $63.3 \pm 2.5$ & $74.8 \pm 2.4$ & $<0.0001$ \\
\hline Non-Hispanic Black (\%) & $15.7 \pm 2.2$ & $10.2 \pm 1.8$ & 0.0001 & $12.1 \pm 1.4$ & $5.5 \pm 1.1$ & $<0.0001$ \\
\hline Non-Hispanic Asian (\%) & $4.6 \pm 0.8$ & $4.6 \pm 0.8$ & 0.9331 & $6.0 \pm 0.8$ & $2.6 \pm 0.5$ & $<0.0001$ \\
\hline \multicolumn{7}{|c|}{ Poverty Income Ratio (PIR) } \\
\hline PIR $<1.35(\%)$ & $35.8 \pm 2.6$ & $31.3 \pm 3.2$ & 0.0403 & $23.8 \pm 1.4$ & $24.1 \pm 2.2$ & 0.8039 \\
\hline $1.35<=\mathrm{PIR}<=1.85(\%)$ & $11.2 \pm 0.9$ & $11.2 \pm 1.7$ & 0.9543 & $10.4 \pm 0.6$ & $10.2 \pm 1.6$ & 0.9070 \\
\hline PIR $>1.85(\%)$ & $53.0 \pm 2.9$ & $57.5 \pm 3.5$ & 0.0913 & $65.9 \pm 1.7$ & $65.7 \pm 3.2$ & 0.9496 \\
\hline \multicolumn{7}{|c|}{ Physical Activity Level } \\
\hline Sedentary & $12.5 \pm 0.9$ & $10.6 \pm 1.5$ & 0.2326 & $22.4 \pm 0.8$ & $18.8 \pm 1.9$ & 0.0618 \\
\hline Moderate & $23.8 \pm 1.2$ & $18.7 \pm 1.3$ & 0.0106 & $36.3 \pm 0.7$ & $31.7 \pm 2.0$ & 0.0268 \\
\hline Vigorous & $63.7 \pm 1.6$ & $70.7 \pm 1.8$ & 0.0019 & $41.2 \pm 0.9$ & $49.4 \pm 2.4$ & 0.0013 \\
\hline \multicolumn{7}{|c|}{ Weight Status } \\
\hline Overweight (\%) & $17.9 \pm 0.6$ & $13.2 \pm 0.9$ & 0.0002 & $32.5 \pm 0.6$ & $33.2 \pm 2.2$ & 0.7501 \\
\hline Overweight or Obese (\%) & $36.7 \pm 1.4$ & $28.3 \pm 1.5$ & $<0.0001$ & $71.9 \pm 0.8$ & $67.0 \pm 1.7$ & 0.0034 \\
\hline Obese (\%) & $18.9 \pm 1.2$ & $15.0 \pm 1.3$ & 0.0082 & $39.5 \pm 0.9$ & $33.8 \pm 2.3$ & 0.0162 \\
\hline
\end{tabular}

${ }^{1}$ Data are means and standard errors using Day 1 dietary recall data. ${ }^{2}$ Below RS: below recommended servings of dairy; ${ }^{3}$ Above RS: at or above recommended servings of dairy. ${ }^{3}$ P-value represents t-test comparing Below RS vs. Above RS. A cup-equivalent of dairy foods is 1 cup of milk, yogurt, or fortified soymilk; 1.5 ounces of natural cheese; or 2 ounces of processed cheese. Poverty Income Ratio (PIR).

Similarly, adults (19+ years) who met dairy food recommendations were more likely to be younger, male, non-Hispanic white, with "vigorous" activity levels and were less likely to have a "moderate" activity level, be non-Hispanic black, overweight or obese. Adults meeting recommendations were also less likely to be non-Hispanic Asian and consumed more energy and food but with a lower BMI. There were no differences in PIR between adults meeting versus not meeting dairy food recommendations (Table 2).

Adults and children (2+ years, females and males) meeting dairy food recommendations were less likely to be below the EAR for calcium, magnesium, phosphorus, protein, riboflavin, vitamin A, vitamin B12, vitamin D, selenium, and zinc than those not meeting dairy food recommendations (Table 2). A larger percentage of the population meeting dairy recommendations was also above the AI for potassium $(71.5 \%)(p<0.0001)$ and choline $(36.9 \%)(p<0.0001)$ compared to those not meeting dairy recommendations. A larger percentage of the population not meeting dairy recommendations 
consumed more than $10 \%$ of calories from added sugars $(64 \%)(p=0.0021)$. Americans meeting dairy recommendations were, however, more likely to exceed $10 \%$ of calories from saturated fat $(p<0.0001)$ and to consume above the AI for sodium $(p<0.0001)$ than those who did not meet dairy recommendations. Population inadequacy (\% below EAR) for calcium, phosphorus, protein, riboflavin, vitamin B12, and zinc was close to zero for the population group meeting dairy food recommendations (Table 4).

Table 3. Energy intake, grams of food intake, and Body Mass Index of U.S. population 2+ years consuming above recommended daily servings of dairy and those consuming fewer than the recommended daily servings of dairy in the National Health and Nutrition Examination Survey $2013-2016^{1}$.

\begin{tabular}{ccc}
\hline Additional Demographics & $\begin{array}{c}\text { Population below } \\
\text { Dairy Recommendations }\end{array}$ & $\begin{array}{c}\text { Population above } \\
\text { Dairy Recommendations }\end{array}$ \\
\hline & Average Energy Intake & \\
\hline Kilocalories (kcal) & $1926 \pm 12$ & \\
\hline Food $(\mathrm{g})$ & Amount of Food Consumed & $3553 \pm 67$ \\
\hline & $3093 \pm 35$ & \\
\hline BMI z-score (ages 2-18 years) & Body Mass Index (BMI) Z-score/BMI & $0.46 \pm 0.04$ \\
BMI (ages 19+ years) & $0.59 \pm 0.04$ & $28.3 \pm 0.3$ \\
\hline${ }^{1}$ Data are means and standard errors using Day 1 dietary recall data. Body Mass Index (BMI).
\end{tabular}

Table 4. Percent U.S. population (ages 2+, male and female combined) below the Estimated Average Requirement or Above Adequate Intake among those consuming above recommended daily servings of dairy and those consuming fewer than the recommended daily servings of dairy in the National Health and Nutrition Examination Survey 2013-2016 ${ }^{1}$.

\begin{tabular}{cccc}
\hline & \multicolumn{2}{c}{ \% Population below Estimated Average Requirement } \\
\cline { 2 - 4 } Nutrients & $\begin{array}{c}\text { Below Dairy } \\
\text { Recommendations } \\
(\boldsymbol{n}=\mathbf{1 3}, \mathbf{1 4 6})\end{array}$ & $\begin{array}{c}\text { Above Dairy } \\
\text { Recommendations } \\
(\boldsymbol{n}=\mathbf{2 6 3 6 )}\end{array}$ & $\boldsymbol{p}$-Value $\mathbf{~}^{2}$ \\
\hline Calcium & $63.3 \pm 0.8$ & $0.05 \pm 0.07$ & $<0.0001$ \\
Magnesium & $57.1 \pm 1.1$ & $14.4 \pm 1.2$ & $<0.0001$ \\
Phosphorus & $6.5 \pm 0.6$ & $0.01 \pm 0.01$ & $<0.0001$ \\
Protein & $2.3 \pm 0.4$ & $0.0 \pm 0.0$ & $<0.0001$ \\
Riboflavin & $3.5 \pm 0.4$ & $0.01 \pm 0.004$ & $<0.0001$ \\
Vitamin A & $50.9 \pm 1.6$ & $3.4 \pm 1.2$ & $<0.0001$ \\
Vitamin B12 & $5.5 \pm 0.7$ & $0.01 \pm 0.02$ & $<0.0001$ \\
Vitamin D (D2 + D3) & $98.5 \pm 0.3$ & $59.7 \pm 2.3$ & $<0.0001$ \\
Selenium & $0.6 \pm 0.2$ & $0.0 \pm 0.0$ & $<0.0001$ \\
Zinc & $22.0 \pm 1.1$ & $0.2 \pm 0.1$ & $<0.0001$ \\
& $\%$ Population above Adequate Intake/Recommended Intakes \\
\hline Sodium & $98.9 \pm 0.2$ & $100 \pm 0.0$ & $<0.0001$ \\
Potassium & $21.2 \pm 0.9$ & $71.5 \pm 2.0$ & $<0.0001$ \\
Choline & $6.5 \pm 0.6$ & $36.9 \pm 2.3$ & $<0.0001$ \\
Energy from saturated & $65.8 \pm 1.4$ & $98.1 \pm 0.7$ & 0.0021 \\
fat & & &
\end{tabular}

1 Data are means and standard errors using usual intake estimated with the National Cancer Institute method. $2 p$-value assessed using z-score comparing percentages for below dairy recommendations to percentages for at/above dairy recommendations. 
When separated by age (children 2-18 years and adults 19+ years), a smaller percentage of children and adults meeting dairy food recommendations was below the EAR for calcium $(p<0.0001)$, magnesium $(p<0.0001)$, phosphorus $(p<0.0001)$, protein (adults only) $(p<0.0001)$, riboflavin $(p<0.0001)$, vitamin A $(p<0.0001)$, vitamin B12 $(p=0.0084$ for children; $p<0.0001$ for adults $)$, vitamin $\mathrm{D}(p<0.0001)$, and zinc $(p<0.001)$ compared to adults and children who did not meet dairy recommendations. In addition, a higher percentage of children and adults meeting dairy food recommendations was above AI for potassium $(p<0.0001)$ and choline $(p<0.0001)$ compared to those not meeting recommendations. Inadequate intake of calcium, phosphorus, protein, riboflavin, and vitamin B12 was close to zero (\% below EAR) for both children and adults meeting dairy food recommendations (Table 5). More children and adults consuming below recommendations for dairy food intake exceeded added sugar intakes ( $p<0.0001$ for children; $p=0.0075$ for adults), while adults and children meeting dairy recommendations were more likely to exceed the recommended intakes for saturated fat $(p<0.0001)$ and sodium ( $p=0.0343$ for children; $p<0.0001$ for adults).

Table 5. Percent U.S. population (by age group) below the Estimated Average Requirement or Above Adequate Intake among those consuming above recommended daily servings of dairy (Above RS) and below recommended daily servings of dairy (Below RS), in the National Health and Nutrition Examination Survey 2013-2016 ${ }^{1}$.

\begin{tabular}{|c|c|c|c|c|c|c|}
\hline \multirow{3}{*}{ Nutrients } & \multicolumn{6}{|c|}{ \% Population below Estimated Average Requirement } \\
\hline & \multicolumn{3}{|c|}{ Children 2-18 Years } & \multicolumn{3}{|c|}{ Adults $19+$ Years } \\
\hline & $\begin{array}{c}\text { Below RS }{ }^{2} \\
(n=4212)\end{array}$ & $\begin{array}{c}\text { Above RS }^{3} \\
(n=1458)\end{array}$ & $p$-Value ${ }^{4}$ & $\begin{array}{l}\text { Below RS } \\
(n=8934)\end{array}$ & $\begin{array}{l}\text { Above RS } \\
(n=1178)\end{array}$ & $p$-Value \\
\hline Calcium & $77.0 \pm 1.2$ & $0.1 \pm 0.2$ & $<0.0001$ & $59.6 \pm 1.0$ & $0.01 \pm 0.01$ & $<0.0001$ \\
\hline Magnesium & $45.3 \pm 1.5$ & $10.8 \pm 1.8$ & $<0.0001$ & $60.1 \pm 1.2$ & $16.8 \pm 1.9$ & $<0.0001$ \\
\hline Phosphorus & $29.2 \pm 2.4$ & $0.03 \pm 0.04$ & $<0.0001$ & $0.8 \pm 0.2$ & $0.0 \pm 0.0$ & $<0.0001$ \\
\hline Protein & $1.5 \pm 0.8$ & $0.0 \pm 0.0$ & 0.0615 & $2.5 \pm 0.4$ & $0.0 \pm 0.0$ & $<0.0001$ \\
\hline Riboflavin & $1.9 \pm 0.8$ & $0.0 \pm 0.0$ & 0.0179 & $3.8 \pm 0.4$ & $0.01 \pm 0.01$ & $<0.0001$ \\
\hline Vitamin A & $38.5 \pm 2.5$ & $0.3 \pm 0.2$ & $<0.0001$ & $54.1 \pm 1.5$ & $5.4 \pm 1.9$ & $<0.0001$ \\
\hline Vitamin B12 & $2.6 \pm 1.0$ & $0.0 \pm 0.0$ & 0.0084 & $6.9 \pm 0.8$ & $0.01 \pm 0.03$ & $<0.0001$ \\
\hline Vitamin D & $99.5 \pm 0.2$ & $57.4 \pm 2.8$ & $<0.0001$ & $98.3 \pm 0.4$ & $60.8 \pm 2.9$ & $<0.0001$ \\
\hline Selenium & $0.2 \pm 0.2$ & $0.0 \pm 0.0$ & 0.24 & $0.7 \pm 0.2$ & $0.0 \pm 0.0$ & $<0.0001$ \\
\hline \multirow[t]{2}{*}{ Zinc } & $17.7 \pm 2.2$ & $0.1 \pm 0.1$ & $<0.0001$ & $23.0 \pm 1.1$ & $0.3 \pm 0.2$ & $<0.0001$ \\
\hline & \multicolumn{6}{|c|}{ \% Population above Adequate Intake/Recommended Intakes } \\
\hline Sodium & $99.7 \pm 0.2$ & $100 \pm 0.0$ & 0.0343 & $98.8 \pm 0.2$ & $100 \pm 0.01$ & $<0.0001$ \\
\hline Potassium & $14.0 \pm 1.6$ & $70.3 \pm 2.7$ & $<0.0001$ & $23.1 \pm 1.1$ & $72.1 \pm 2.5$ & $<0.0001$ \\
\hline Choline & $10.6 \pm 1.3$ & $48.4 \pm 2.6$ & $<0.0001$ & $5.5 \pm 0.7$ & $30.2 \pm 2.7$ & $<0.0001$ \\
\hline $\begin{array}{l}\text { Energy from } \\
\text { saturated fat }\end{array}$ & $72.2 \pm 2.9$ & $98.3 \pm 1.1$ & $<0.0001$ & $64.0 \pm 1.7$ & $98.0 \pm 1.0$ & $<0.0001$ \\
\hline $\begin{array}{c}\text { Energy from } \\
\text { added } \\
\text { sugars }\end{array}$ & $82.5 \pm 2.7$ & $64.6 \pm 2.8$ & $<0.0001$ & $59.4 \pm 1.0$ & $52.0 \pm 2.6$ & 0.0075 \\
\hline
\end{tabular}

A significantly smaller percentage of both females and males meeting dairy food recommendations was below the EAR for calcium $(p<0.0001)$, magnesium $(p<0.0001)$, phosphorus $(p<0.0001)$, protein $(p<0.0001)$, riboflavin $(p<0.0001)$, vitamin A $(p<0.0001)$, vitamin B12 $(p<0.0001)$, vitamin D ( $p<0.0001)$, selenium ( $p=0.0001$ for females; $p=0.0246$ for males), and zinc $(p<0.0001)$ compared to those who did not meet dairy recommendations (Table 6). Furthermore, a larger percentage of females and males meeting dairy food recommendations was above the AI for potassium and choline compared to those not meeting recommendations ( $p<0.0001$ for both). Population inadequacy ( $\%$ below EAR) for calcium, phosphorus, protein, riboflavin, selenium, zinc, and vitamin B12 was also 
close to zero for both females and males meeting dairy food recommendations. Females and males meeting dairy food recommendations were more likely to exceed $10 \%$ of calories from saturated fat $(p<0.0001)$. Females meeting dairy recommendations were less likely to exceed 10\% of energy from added sugars than females consuming below recommendations for dairy food intake $(p=0.0003)$.

Table 6. Percent U.S. population (by sex) below the Estimated Average Requirement or Above Adequate Intake among those consuming above recommended daily servings of dairy (Above RS) and below recommended daily servings of dairy (Below RS), ages 2+, in the National Health and Nutrition Examination Survey 2013-2016 ${ }^{1}$.

\begin{tabular}{|c|c|c|c|c|c|c|}
\hline \multirow{3}{*}{ Nutrients } & \multicolumn{6}{|c|}{ \% Population below Estimated Average Requirement } \\
\hline & \multicolumn{3}{|c|}{ Females } & \multicolumn{3}{|c|}{ Males } \\
\hline & $\begin{array}{c}\text { Below RS }{ }^{2} \\
n=6969\end{array}$ & $\begin{array}{c}\text { Above RS } \\
n=1002\end{array}$ & $p$-Value ${ }^{4}$ & $\begin{array}{c}\text { Below RS } \\
n=6177\end{array}$ & $\begin{array}{c}\text { Above RS } \\
n=1634\end{array}$ & $p$-Value \\
\hline Calcium & $73.5 \pm 0.9$ & $0.1 \pm 0.1$ & $<0.0001$ & $51.5 \pm 1.2$ & $0.02 \pm 0.02$ & $<0.0001$ \\
\hline Magnesium & $54.8 \pm 1.3$ & $11.5 \pm 2.0$ & $<0.0001$ & $60.0 \pm 1.3$ & $16.1 \pm 1.4$ & $<0.0001$ \\
\hline Phosphorus & $8.5 \pm 0.7$ & $0.03 \pm 0.03$ & $<0.0001$ & $4.3 \pm 0.6$ & $0.01 \pm 0.01$ & $<0.0001$ \\
\hline Protein & $2.8 \pm 0.5$ & $0.0 \pm 0.0$ & $<0.0001$ & $1.6 \pm 0.3$ & $0.0 \pm 0.01$ & $<0.0001$ \\
\hline Riboflavin & $3.3 \pm 0.5$ & $0.0 \pm 0.01$ & $<0.0001$ & $3.6 \pm 0.6$ & $0.0 \pm 0.01$ & $<0.0001$ \\
\hline Vitamin A & $45.1 \pm 1.9$ & $1.7 \pm 1.0$ & $<0.0001$ & $57.6 \pm 1.7$ & $4.3 \pm 1.6$ & $<0.0001$ \\
\hline Vitamin B12 & $8.4 \pm 1.2$ & $0.02 \pm 0.04$ & $<0.0001$ & $2.4 \pm 0.5$ & $0.0 \pm 0.01$ & $<0.0001$ \\
\hline Vitamin D & $99.3 \pm 0.2$ & $67.7 \pm 3.5$ & $<0.0001$ & $97.6 \pm 0.6$ & $55.0 \pm 2.7$ & $<0.0001$ \\
\hline Selenium & $1.1 \pm 0.3$ & $0.0 \pm 0.0$ & 0.0001 & $0.1 \pm 0.1$ & $0.0 \pm 0.0$ & 0.0246 \\
\hline \multirow[t]{2}{*}{ Zinc } & $21.6 \pm 1.4$ & $0.09 \pm 0.09$ & $<0.0001$ & $22.4 \pm 1.4$ & $0.3 \pm 0.2$ & $<0.0001$ \\
\hline & \multicolumn{6}{|c|}{$\%$ Population above Adequate Intake } \\
\hline Sodium & $98.1 \pm 0.3$ & $100 \pm 0.01$ & $<0.0001$ & $99.9 \pm 0.03$ & $100 \pm 0.0$ & 0.0517 \\
\hline Potassium & $22.9 \pm 1.2$ & $70.4 \pm 2.7$ & $<0.0001$ & $19.3 \pm 1.0$ & $71.7 \pm 2.3$ & $<0.0001$ \\
\hline Choline & $4.7 \pm 0.5$ & $33.5 \pm 2.9$ & $<0.0001$ & $8.7 \pm 1.0$ & $39.2 \pm 2.9$ & $<0.0001$ \\
\hline $\begin{array}{l}\text { Energy from } \\
\text { saturated fat }\end{array}$ & $65.5 \pm 1.9$ & $99.3 \pm 0.6$ & $<0.0001$ & $66.0 \pm 1.8$ & $97.5 \pm 1.0$ & $<0.0001$ \\
\hline $\begin{array}{c}\text { Energy from } \\
\text { added } \\
\text { sugars }\end{array}$ & $64.9 \pm 1.4$ & $54.0 \pm 2.7$ & 0.0003 & $63.0 \pm 1.2$ & $58.3 \pm 2.8$ & 0.1185 \\
\hline
\end{tabular}

\footnotetext{
${ }^{1}$ Data are means and standard errors using usual intake estimated with the National Cancer Institute method.

2 Below RS: below recommended servings of dairy ${ }^{3}$ Above RS: at or above recommended servings of dairy 4 -value assessed using z-score comparing percentages for below dairy recommendations to percentages for at/above dairy recommendations.
}

Results were similar when nutrient intake and dairy food intake were assessed by ethnicity and race (Table 7). A smaller percentage of Hispanics, non-Hispanic Blacks, non-Hispanic Whites, and non-Hispanic Asians meeting dairy recommendations was below EAR for calcium $(p<0.0001)$, magnesium $(p<0.0001)$, phosphorus $(0.0016$ for non-Hispanic Asians; $p<0.0001$ for all other groups), protein ( $p=0.0008$ for Hispanics; $p=0.0114$ for non-Hispanic Asians; $p<0.0001$ for both other groups), riboflavin ( $p=0.0002$ for non-Hispanic Asians; $p<0.0001$ for all other groups), vitamin $\mathrm{A}(p<0.0001)$, vitamin B12 ( $p=0.0003$ for non-Hispanic Blacks; $p=0.0001$ for Hispanics; $p<0.0001$ for both other groups), vitamin $\mathrm{D}(p<0.0001)$, and zinc $(p<0.0001)$ compared to those not meeting dairy recommendations. A larger percentage of Hispanics, non-Hispanic Blacks, non-Hispanic Whites and non-Hispanic Asians meeting dairy food recommendations was also above AI for potassium and choline $(p<0.0001)$ compared to individuals from those same groups not meeting dairy food recommendations. Population inadequacy (\% below EAR) for calcium, phosphorus, protein, riboflavin, vitamin B12, and zinc was close to zero for Hispanics, non-Hispanic Blacks, non-Hispanic Whites, and non-Hispanic Asians meeting dairy food recommendations. Hispanics and non-Hispanic Whites meeting dairy recommendations were more likely to exceed the AI for sodium ( $p<0.0001)$, and Americans across racial and ethnic groups who met dairy recommendations exceeded $10 \%$ of calories from saturated fats $(p<0.0001)$. 
Table 7. Percent U.S. population (by ethnicity/race) below the Estimated Average Requirement or Above Adequate Intake among those consuming above recommended daily servings of dairy (Above RS) and below recommended daily servings of dairy (Below RS), ages 2+, in the National Health and Nutrition Examination Survey $2013-2016^{1}$

\begin{tabular}{|c|c|c|c|c|c|c|c|c|c|c|c|c|}
\hline \multirow{3}{*}{ Nutrients } & \multicolumn{12}{|c|}{ \% Population below Estimated Average Requirement } \\
\hline & \multicolumn{3}{|c|}{ Hispanics } & \multicolumn{3}{|c|}{ Non-Hispanic Blacks } & \multicolumn{3}{|c|}{ Non-Hispanic Whites } & \multicolumn{3}{|c|}{ Non-Hispanic Asians } \\
\hline & $\begin{array}{l}\text { Below RS } \\
2 n=3787\end{array}$ & $\begin{array}{c}\text { Above RS } \\
{ }^{3} n=811\end{array}$ & $p$-Value ${ }^{4}$ & $\begin{array}{c}\text { Below RS } \\
n=3035\end{array}$ & $\begin{array}{c}\text { Above RS } \\
n=404\end{array}$ & $p$-Value & $\begin{array}{c}\text { Below RS } \\
n=4430\end{array}$ & $\begin{array}{c}\text { Above RS } \\
n=1089\end{array}$ & $p$-Value & $\begin{array}{c}\text { Below RS } \\
n=1325\end{array}$ & $\begin{array}{c}\text { Above RS } \\
n=183\end{array}$ & $p$-Value \\
\hline Calcium & $58.6 \pm 1.3$ & $0.0 \pm 0.03$ & $<0.0001$ & $74.3 \pm 1.7$ & $0.0 \pm 0.7$ & $<0.0001$ & $61.6 \pm 1.1$ & $0.05 \pm 0.07$ & $<0.0001$ & $76.2 \pm 3.8$ & $0.01 \pm 0.2$ & $<0.0001$ \\
\hline Magnesium & $52.7 \pm 1.8$ & $12.4 \pm 2.0$ & $<0.0001$ & $66.6 \pm 1.3$ & $14.3 \pm 2.3$ & $<0.0001$ & $57.9 \pm 1.5$ & $15.0 \pm 1.6$ & $<0.0001$ & $49.4 \pm 3.0$ & $10.6 \pm 3.7$ & $<0.0001$ \\
\hline Phosphorus & $7.7 \pm 0.9$ & $0.01 \pm 0.01$ & $<0.0001$ & $9.9 \pm 0.8$ & $0.0 \pm 0.01$ & $<0.0001$ & $5.5 \pm 0.7$ & $0.01 \pm 0.03$ & $<0.0001$ & $4.0 \pm 1.3$ & $0.01 \pm 0.08$ & 0.0016 \\
\hline Protein & $1.6 \pm 0.5$ & $0.0 \pm 0.01$ & 0.0008 & $3.6 \pm 0.7$ & $0.0 \pm 0.0$ & $<0.0001$ & $2.4 \pm 0.6$ & $0.0 \pm 0.0$ & $<0.0001$ & $1.3 \pm 0.4$ & $0.05 \pm 0.2$ & 0.0114 \\
\hline Riboflavin & $4.5 \pm 0.9$ & $0.0 \pm 0.0$ & $<0.0001$ & $7.0 \pm 1.2$ & $0.0 \pm 0.01$ & $<0.0001$ & $2.0 \pm 0.4$ & $0.02 \pm 0.02$ & $<0.0001$ & $5.1 \pm 1.4$ & $0.0 \pm 0.02$ & 0.0002 \\
\hline Vitamin A & $58.5 \pm 2.3$ & $1.0 \pm 1.0$ & $<0.0001$ & $59.3 \pm 2.1$ & $2.8 \pm 2.3$ & $<0.0001$ & $47.0 \pm 2.3$ & $4.3 \pm 1.7$ & $<0.0001$ & $48.8 \pm 2.9$ & $0.5 \pm 2.2$ & $<0.0001$ \\
\hline $\begin{array}{l}\text { Vitamin } \\
\text { B12 }\end{array}$ & $6.4 \pm 1.6$ & $0.02 \pm 0.05$ & 0.0001 & $4.7 \pm 1.3$ & $0.0 \pm 0.01$ & 0.0003 & $5.2 \pm 1.0$ & $0.01 \pm 0.02$ & $<0.0001$ & $11.5 \pm 2.3$ & $0.0 \pm 0.0$ & $<0.0001$ \\
\hline Vitamin D & $98.9 \pm 0.3$ & $65.1 \pm 6.5$ & $<0.0001$ & $99.6 \pm 0.2$ & $69.1 \pm 5.1$ & $<0.0001$ & $98.4 \pm 0.4$ & $58.2 \pm 3.1$ & $<0.0001$ & $95.5 \pm 1.3$ & $40.8 \pm 12.1$ & $<0.0001$ \\
\hline Selenium & $0.5 \pm 0.2$ & $0.0 \pm 0.0$ & 0.0039 & $1.0 \pm 0.4$ & $0.0 \pm 0.0$ & 0.0143 & $0.8 \pm 0.2$ & $0.0 \pm 0.0$ & 0.0003 & $0.1 \pm 0.1$ & $0.03 \pm 0.06$ & 0.5076 \\
\hline \multirow[t]{2}{*}{ Zinc } & $18.7 \pm 2.0$ & $0.08 \pm 0.1$ & $<0.0001$ & $27.6 \pm 1.6$ & $0.4 \pm 0.2$ & $<0.0001$ & $22.1 \pm 1.7$ & $0.3 \pm 0.2$ & $<0.0001$ & $20.0 \pm 3.1$ & $0.6 \pm 1.3$ & $<0.0001$ \\
\hline & \multicolumn{12}{|c|}{$\%$ Population above Adequate Intake } \\
\hline Sodium & $99.0 \pm 0.2$ & $100 \pm 0.0$ & $<0.0001$ & $99.5 \pm 1.5$ & $100 \pm 0.02$ & 0.7372 & $98.9 \pm 0.3$ & $100 \pm 0.01$ & $<0.0001$ & $99.8 \pm 0.2$ & $99.6 \pm 0.7$ & 0.8152 \\
\hline Potassium & $22.7 \pm 1.6$ & $72.4 \pm 3.2$ & $<0.0001$ & $10.6 \pm 3.5$ & $90.1 \pm 16.0$ & $<0.0001$ & $19.7 \pm 3.2$ & $71.0 \pm 2.7$ & $<0.0001$ & $24.8 \pm 2.7$ & $83.2 \pm 8.5$ & $<0.0001$ \\
\hline Choline & $8.6 \pm 0.9$ & $46.8 \pm 3.7$ & $<0.0001$ & $7.8 \pm 1.1$ & $51.7 \pm 10.0$ & $<0.0001$ & $5.8 \pm 0.9$ & $32.7 \pm 3.1$ & $<0.0001$ & $7.6 \pm 1.5$ & $52.7 \pm 8.1$ & $<0.0001$ \\
\hline $\begin{array}{c}\text { Energy } \\
\text { from } \\
\text { saturated } \\
\text { fat }\end{array}$ & $58.6 \pm 2.5$ & $98.4 \pm 1.7$ & $<0.0001$ & $60.9 \pm 3.1$ & $99.1 \pm 1.3$ & $<0.0001$ & $72.3 \pm 2.3$ & $98.3 \pm 0.9$ & $<0.0001$ & $31.1 \pm 2.2$ & $80.5 \pm 6.7$ & $<0.0001$ \\
\hline $\begin{array}{c}\text { Energy } \\
\text { from } \\
\text { added } \\
\text { sugars }\end{array}$ & $68.1 \pm 1.8$ & $49.5 \pm 3.7$ & $<0.0001$ & $76.1 \pm 1.9$ & $73.3 \pm 4.6$ & 0.5759 & $63.0 \pm 1.6$ & $56.8 \pm 2.5$ & 0.0346 & $28.8 \pm 2.7$ & $43.2 \pm 5.0$ & 0.0102 \\
\hline
\end{tabular}

${ }^{1}$ Data are means and standard errors using usual intake estimated with the National Cancer Institute method. ${ }^{2}$ Below RS: below recommended servings of dairy; ${ }^{3}$ Above RS: at or above recommended servings of dairy ${ }^{4} p$-value assessed using z-score comparing percentages for below dairy recommendations to percentages for at/above dairy recommendations. 


\section{Discussion}

The percentage of the American population meeting dairy recommendations was more likely to be above the EAR for a range of nutrients and the AI for potassium and choline. Most children and adults in this study who met recommendations for dairy foods had intakes above the EAR for calcium, magnesium, phosphorus, protein, riboflavin, vitamin A, and vitamin B12, and over 70\% of Americans who met recommendations for dairy foods also met the AI for potassium. The nutrient adequacy results were similar across age, sex, and ethnic/racial groups. American adults meeting dairy food recommendations were also more likely to have adequate intake of protein than adults not meeting dairy recommendations, though this difference was not significant in children. Population inadequacy (\% below EAR) was close to zero for calcium, phosphorus, riboflavin, and vitamin B12 among Americans meeting dairy food recommendations across all age, sex, and ethnic/racial groups. In contrast, nearly $60 \%$ of Americans ages 2 years and older who did not meet dairy food recommendations consumed calcium and magnesium below the EAR, and only $20 \%$ of Americans not meeting dairy recommendations were above the AI for potassium [17]. Results of this study indicate that it can be difficult to meet nutrient needs without adequate consumption of dairy foods.

In addition to being recommended by the DGA, dairy foods are part of healthy eating patterns recommended by the American College of Cardiology and the American Heart Association [18-20], the National Osteoporosis Foundation [21], and the American Academy of Pediatrics [22]. Results of this study indicate that the U.S. population still largely does not meet recommendations for dairy food intake. Some groups especially at risk for underconsuming dairy foods include females (children and adults), older children (9-18 years), older adults, non-Hispanic blacks, non-Hispanic Asian adults, children below a poverty-income ratio of 1.35 , and Americans with overweight or obesity.

Actual or perceived lactose intolerance may be a reason that some Americans, including non-Hispanic blacks and non-Hispanic Asians, do not meet recommendations for dairy intake [23]. A consensus statement published following a 2010 National Institutes of Health Conference on Lactose Intolerance and Health indicates that individuals with lactose intolerance, whether actual or perceived, may be predisposed to "decreased bone accrual, osteoporosis, and other adverse health outcomes" as a result of insufficient dairy food and calcium intake [6]. A 2013 joint consensus statement on lactose intolerance from the National Medical Association and the National Hispanic Medical Association further indicated that some minority groups consume fewer dairy foods than the general population and are at a higher risk for developing chronic disease conditions such as hypertension and diabetes [24], which are associated with low calcium intake from dairy foods [25]. However, most individuals can continue to consume dairy foods while avoiding symptoms of lactose intolerance. A systematic review indicates that individuals with lactose intolerance can tolerate about $12 \mathrm{~g}$ of lactose-the amount in one cup of milk-especially when it is consumed with other foods [26,27]. Management strategies, such as selecting lactose-free milk and choosing yogurt with live and active cultures, can also help people with lactose intolerance to meet recommendations for dairy intake $[6,27,28]$. Lactose-free cow's milk is an increasingly popular option for individuals with lactose intolerance, is recommended in the 2015 DGA as an option for meeting dairy recommendations, and can be found in $18 \%$ of U.S. households and $96 \%$ of U.S. retail food outlets [29-31].

Though dairy foods are a low-cost source of important shortfall nutrients for Americans [32], cost may also be a factor contributing to different nutrient intake among some groups. Children from households with a PIR $<1.35$ were more likely to consume fewer servings of dairy foods than the DGA recommends. While the DGA takes many factors into account when developing healthy eating patterns, diet cost and food accessibility, including food cost, are not part of those considerations. While over $94 \%$ of retail outlets (food stores, drugstores, and convenience stores) in the U.S. sell one or more types of dairy food and most households with children purchase some dairy foods [33], cost and perishability of dairy foods may still prohibit some families from purchasing adequate amounts of dairy foods to enable all family members to meet recommendations. Market research data from early 2020 (through 23 February 2020) [34] indicate that a serving of low-fat milk may cost as little USD 0.18 from a private 
label gallon container, but the cost rises to USD 0.66 per serving, on average, for low-fat milk purchased by the half gallon. In addition, individuals needing lactose-free or reduced-lactose dairy products may also have challenges accessing adequate dairy foods, as these products also tend to be more expensive [34]. While dairy foods may be physically accessible and a low-cost source for important nutrients, they may not be sufficiently financially accessible for some Americans-an obstacle to adequate intake.

Americans with overweight or obesity are also at higher risk of underconsuming dairy foods, according to results of this study. While there are many potential reasons that this group may not be meeting recommendations for dairy intake, one reason may be due to a perception that consuming dairy foods is linked with increased adiposity. In this study, children and adults meeting dairy food recommendations tended to report higher energy intakes but lower BMI z-scores or BMIs. This finding aligns with several other studies in the literature, indicating that consuming dairy foods in recommended amounts is not linked with increased risk of adiposity in children or adults. For instance, two meta-analyses [35,36] and a systematic review [37] as well as several prospective cohort studies and cross sectional studies [38-42] indicate that dairy intake is inversely associated with or not associated with markers of adiposity in children and adolescents. In those reviews and studies, drinking plain milk was not associated with BMI or BMI z-scores in children, though it increased caloric intake and energy density at meals. A systematic review [36] and a randomized controlled trial [43] also indicate that drinking milk is not linked to increased risk of overweight and obesity in adults. Finally, the Scientific Report of the 2020-2025 Dietary Guidelines Advisory Committee (DGAC) notes that consuming low-fat dairy foods as part of a healthy eating pattern has beneficial impacts on outcomes related to growth, size, body composition, and obesity in adults (moderate evidence) and children (limited evidence) [3]. The Scientific Report of the 2020 DGAC also found limited evidence to suggest that milk intake was not associated with adiposity in either children or adults [3].

This study also indicates that Americans who consumed adequate amounts of dairy foods were more likely to consume more sodium and calories from saturated fat and, for some groups, fewer calories from added sugars. The average sodium intake of Americans ages 2+, according to a publication from the National Academies of Science, Engineering, and Medicine, was approximately $3400 \mathrm{mg} /$ day, which exceeds the sodium AI (1500 mg/day for ages 14+) [44]. In this study, Americans ages 2+ who did not meet dairy food recommendations consumed less sodium, averaging approximately $3200 \mathrm{mg} /$ day of sodium compared to the over $4400 \mathrm{mg} /$ day consumed by Americans who did meet dairy food recommendations. The only groups in this study without significant differences in percent population meeting dairy recommendations and exceeding the AI for sodium were males ( $p=0.0517)$, non-Hispanic blacks $(p=0.7372)$, and non-Hispanic Asians $(p=0.8152)$. In this study, individuals meeting dairy recommendations were more likely to consume above $10 \%$ of their calories from saturated fat regardless of age (children, adults), gender (females and males), and racial or ethnic background (Hispanics, non-Hispanic Blacks, non-Hispanic Whites, non-Hispanic Asians).

The relationship between meeting dairy recommendations and added sugar intakes was less consistent. Among all Americans over the age of 2, more individuals consuming less than recommended dairy intakes consumed more than $10 \%$ of their calories from added sugars $(p=0.0021)$. This association was not significant across all population segments. Hispanics, non-Hispanic Whites, and non-Hispanic Asians, females, children, and adults had significant relationships between added sugars intake and dairy intake. Children and non-Hispanic Asians meeting dairy recommendations were more likely to exceed $10 \%$ of calories from added sugars, but for all other population groups, those meeting dairy recommendations were less likely to exceed $10 \%$ of calories from added sugars. There was not a significant difference in the percent of the population exceeding added sugar recommendations between those meeting and those not meeting dairy recommendations among males and non-Hispanic Blacks.

The relationships between adequate dairy intake and elevated consumption of sodium and saturated fats and, for some population groups, added sugars, observed in this study, indicate a need for consumers to receive additional education around DGA recommendations. Consuming 
adequate servings of dairy foods while staying within recommended limits for sodium, added sugars, and saturated fat is possible, as demonstrated in food pattern modeling presented in both the 2015 DGA [1] and the Scientific Report of the 2020 Dietary Guidelines Advisory Committee [3]. Consumers may need additional guidance to follow the recommended healthy eating patterns and implement recommendations in the DGA.

Finally, while this study used a large nationally representative data sample, it also had some important limitations. Some limitations include the cross-sectional study design of NHANES, which prevents the determination of any cause and effect relationship. In addition, NHANES relies on self-reported dietary intake date, which may over- or underrepresent actual intake on an individual basis. It is also difficult to completely account for residual confounding (e.g., those who met recommendations might have also consumed more fruits and vegetables or other types of foods); thus, the improvements in nutrient intakes cannot be ascribed to dairy foods alone. Furthermore, the data used in this study were limited to foods available in NHANES 2013-2016, which may not reflect all food options currently in the marketplace, and we did not attempt to characterize the food source of the dairy intake, which may have been milk, cheese, yogurt, or other dairy foods, which could impact the results.

\section{Conclusions}

Although it may be possible for Americans to meet their nutrient needs without dairy foods, evidence from NHANES about how Americans actually eat indicates that dairy foods make important and unique contributions to dietary patterns, contributing calcium, magnesium, phosphorus, riboflavin, vitamin A, vitamin B12, zinc, potassium, and choline. Those who are not eating enough dairy foods are not consuming sufficient amounts of these nutrients from other sources. Those Americans who do meet current recommendations for dairy food intake have improved nutrient adequacy for several important shortfall nutrients.

Author Contributions: Conceptualization, C.J.C. and V.L.F.III; methodology, J.M.H., C.J.C., V.L.F.III; writing-original draft preparation, J.M.H.; writing - review and editing, C.J.C. and V.L.F.III All authors have read and agreed to the published version of the manuscript.

Funding: This study and the writing of this manuscript were supported by the National Dairy Council.

Conflicts of Interest: J.M.H. and C.J.C. work for the National Dairy Council. V.L.F.III, as senior vice-president of Nutrition Impact L.L.C., performs consulting and database analyses for various food and beverage companies and related entities including the National Dairy Council.

\section{References}

1. USDA. HHS 2015-2020 Dietary Guidelines. Available online: http://health.gov/dietaryguidelines/2015/ guidelines/ (accessed on 8 January 2016).

2. USDA. HHS Scientific Report of the 2015 Dietary Guidelines Advisory Committee; U.S. Department of Agriculture: Washington, DC, USA, 2015. Available online: https://health.gov/sites/default/files/2019-09/Scientific-Reportof-the-2015-Dietary-Guidelines-Advisory-Committee.pdf (accessed on 23 February 2020).

3. Dietary Guidelines Advisory Committee. Scientific Report of the 2020 Dietary Guidelines Advisory Committee: Advisory Report to the Secretary of Agriculture and the Secretary of Health and Human Services; U.S. Department of Agriculture: Washington, DC, USA, 2020. Available online: https://www.dietaryguidelines.gov/sites/default/ files/2020-07/ScientificReport_of_the_2020DietaryGuidelinesAdvisoryCommittee_first-print.pdf (accessed on 15 July 2020).

4. Donovan, S.M. National Academies of Sciences, Engineering, and Medicine. Finding a Path to Safety in Food Allergy; Stallings, V.A., Oria, M.P., Eds.; National Academies Press: Washington, DC, USA, 2017. [CrossRef]

5. Savage, J.; Sicherer, S.; Wood, R. The Natural History of Food Allergy. J. Allergy Clin. Immunol. Pract. 2016, 4, 196-203. [CrossRef] [PubMed]

6. Suchy, F.; Brannon, P.; Carpenter, T.; Fernandez, J.; Gilsanz, V.; Gould, J.; Hall, K.; Hui, S.; Lupton, J.; Mennella, J.; et al. NIH Lactose Intolerance Development Conference Statement: Lactose Intolerance and Health. NIH Consens. State Sci. Conf. Statement 2010, 27, 1-27. [CrossRef] 
7. Nicklas, T.A.; Qu, H.; Hughes, S.O.; He, M.; Wagner, S.E.; Foushee, H.R.; Shewchuk, R.M. Self-perceived lactose intolerance results in lower intakes of calcium and dairy foods and is associated with hypertension and diabetes in adults. Am. J. Clin. Nutr. 2011, 94, 191-198. [CrossRef] [PubMed]

8. NIH. Lactose Intolerance and Health Conference. Available online: https://consensus.nih.gov/2010/lactose. htm (accessed on 29 September 2017).

9. O'Neil, C.E.; Nicklas, T.A.; Fulgoni, V.L. Food Sources of Energy and Nutrients of Public Health Concern and Nutrients to Limit with a Focus on Milk and other Dairy Foods in Children 2 to 18 Years of Age: National Health and Nutrition Examination Survey, 2011-2014. Nutrients 2018, 10, 1050. [CrossRef]

10. O'Neil, C.E.; Keast, D.R.; Fulgoni, V.L.; Nicklas, T.A. Food sources of energy and nutrients among adults in the US: NHANES 2003-2006. Nutrients 2012, 4, 2097-2120. [CrossRef] [PubMed]

11. Quann, E.E.; Fulgoni, V.L.; Auestad, N. Consuming the daily recommended amounts of dairy products would reduce the prevalence of inadequate micronutrient intakes in the United States: Diet modeling study based on NHANES 2007-2010. Nutr. J. 2015, 14, 90. [CrossRef]

12. USDA Agricultural Research Service Nutrient Intakes from Foods and Beverages: Mean Amounts Consumed per Individual, by Gender and Age, in the United States, 2013-2014. Available online: www.ars.usda.gov/ nea/bhnrc/fsrg (accessed on 2 February 2020).

13. U.S. Department of Agriculture, Agricultural Research Service. USDA Food and Nutrient Database for Dietary Studies 2013-2014. Food Surveys Research Group Home Page. 2016. Available online: http://www.ars.usda.gov/nea/bhnrc/fsrg (accessed on 2 February 2020).

14. Bowman, S.; Clemens, J.; Friday, J.; Lynch, K.; Moshfegh, A. Food Patterns Equivalents Database 2013-2014: Methodology and User Guide; Food Surveys Research Group, Beltsville Human Nutrition Research Center, Agricultural Research Service, U.S. Department of Agriculture: Beltsville, MD, USA, 2017. Available online: http://www.ars.usda.gov/nea/bhnrc/fsrg (accessed on 6 May 2019).

15. Bowman, S.; Clemens, J.; Shimizu, M.; Friday, J.; Moshfegh, A. Food Patterns Equivalents Database 2015-2016: Methodology and User Guide; Food Surveys Research Group, Beltsville Human Nutrition Research Center, Agricultural Research Service, U.S. Department of Agriculture: Beltsville, MD, USA, 2018. Available online: http://www.ars.usda.gov/nea/bhnrc/fsrg (accessed on 6 May 2019).

16. National Cancer Institute Usual Dietary Intakes: Analytic Datasets for SAS Macros Epidemiology and Genomics Research Program website. Available online: https://epi.grants.cancer.gov/diet/usualintakes/ dataset.html (accessed on 4 October 2019).

17. National Dairy Council. NHANES 2013-2016. Data Source: Centers for Disease Control and Prevention, National Center for Health Statistics, National Health and Nutrition Examination Survey Data; Centers for Disease Control and Prevention: Hyattsville, MD, USA, 2019.

18. Eckel, R.H.; Jakicic, J.M.; Ard, J.D.; de Jesus, J.M.; Houston Miller, N.; Hubbard, V.S.; Lee, I.-M.; Lichtenstein, A.H.; Loria, C.M.; Millen, B.E.; et al. 2013 AHA/ACC Guideline on Lifestyle Management to Reduce Cardiovascular Risk: A Report of the American College of Cardiology/American Heart Association Task Force on Practice Guidelines. J. Am. Coll. Cardiol. 2014, 63, 2960-2984. [CrossRef]

19. Whelton, P.K.; Carey, R.M.; Aronow, W.S.; Casey, D.E.; Collins, K.J.; Dennison Himmelfarb, C.; DePalma, S.M.; Gidding, S.; Jamerson, K.A.; Jones, D.W.; et al. 2017 ACC/AHA/AAPA/ABC/ACPM/AGS/APhA/ASH/ASPC /NMA/PCNA Guideline for the Prevention, Detection, Evaluation, and Management of High Blood Pressure in Adults: A Report of the American College of Cardiology/American Heart Association Task Force on Clinical Practice Guidelines. Hypertension 2018, 71. [CrossRef]

20. Van Horn, L.; Carson, J.A.S.; Appel, L.J.; Burke, L.E.; Economos, C.; Karmally, W.; Lancaster, K.; Lichtenstein, A.H.; Johnson, R.K.; Thomas, R.J.; et al. Recommended Dietary Pattern to Achieve Adherence to the American Heart Association/American College of Cardiology (AHA/ACC) Guidelines: A Scientific Statement From the American Heart Association. Circulation 2016, 134. [CrossRef]

21. Weaver, C.M.; Gordon, C.M.; Janz, K.F.; Kalkwarf, H.J.; Lappe, J.M.; Lewis, R.; O’Karma, M.; Wallace, T.C.; Zemel, B.S. The National Osteoporosis Foundation's position statement on peak bone mass development and lifestyle factors: A systematic review and implementation recommendations. Osteoporos. Int. 2016, 27, 1281-1386. [CrossRef]

22. American Academy of Pediatrics; Kleinman, R.E.; Greer, F.R. Pediatric Nutrition: Policy of the American Academy of Pediatrics, 7th ed.; American Academy of Pediatrics: Elk Grove Village, IL, USA, 2014; ISBN 9781581108163. 
23. Definition \& Facts for Lactose Intolerance NIDDK. Available online: https://www.niddk.nih.gov/healthinformation/digestive-diseases/lactose-intolerance/definition-facts (accessed on 15 May 2020).

24. Bailey, R.K.; Fileti, C.P.; Keith, J.; Tropez-Sims, S.; Price, W.; Allison-Ottey, S.D. Lactose intolerance and health disparities among African Americans and Hispanic Americans: An updated consensus statement. J. Natl. Med. Assoc. 2013, 105, 112-127. [CrossRef]

25. Jarvis, J.K.; Miller, G.D. Overcoming the barrier of lactose intolerance to reduce health disparities. J. Natl. Med. Assoc. 2002, 94, 55-66. [PubMed]

26. Misselwitz, B.; Butter, M.; Verbeke, K.; Fox, M.R. Update on lactose malabsorption and intolerance: Pathogenesis, diagnosis and clinical management. Gut 2019, 68, 2080-2091. [CrossRef]

27. Shaukat, A.; Levitt, M.D.; Taylor, B.C.; MacDonald, R.; Shamliyan, T.A.; Kane, R.L.; Wilt, T.J. Systematic review: Effective management strategies for lactose intolerance. Ann. Intern. Med. 2010, 152, 797-803. [CrossRef] [PubMed]

28. Savaiano, D.A. Lactose digestion from yogurt: Mechanism and relevance. Am. J. Clin. Nutr. 2014, 99, 1251S-1255S. [CrossRef]

29. Dekker, P.; Koenders, D.; Bruins, M. Lactose-Free Dairy Products: Market Developments, Production, Nutrition and Health Benefits. Nutrients 2019, 11, 551. [CrossRef]

30. Rizzo, P.V.; Harwood, W.S.; Drake, M.A. Consumer desires and perceptions of lactose-free milk. J. Dairy Sci. 2020. [CrossRef]

31. IRI, MULO+C (Multi-Outlets + C-Stores). Based on 4 Weeks Ending 11-3-2019. 2019. Available online: https: //www.iriworldwide.com/en-us/solutions/media/pre-campaign/consumer-insights (accessed on 3 November 2019).

32. Hess, J.M.; Cifelli, C.J.; Agarwal, S.; Fulgoni, V.L. Comparing the cost of essential nutrients from different food sources in the American diet using NHANES 2011-2014. Nutr. J. 2019, 18, 68. [CrossRef]

33. IRI database, MULO+C (Multi-Outlets + C-Stores). Based on Calendar Year 2019 Ending 12-29-19.

Available online: https://www.iriworldwide.com/en-us/solutions/media/media-solutions/consumer (accessed on 28 September 2020).

34. IRI Dairy Management Incorporated Custom Database, Multi Outlet + Conv. 2020 through 2/23/20. Milk Prices Based on Gallon Equivalents-National Average. Available online: https://www.iriworldwide. com/en-us/insights/webinars/strategies-for-dairy-success (accessed on 23 February 2020).

35. Kang, K.; Sotunde, O.F.; Weiler, H.A. Effects of milk and milk-product consumption on growth among children and adolescents aged 6-18 years: A meta-analysis of randomized controlled trials. Adv. Nutr. 2019, 10, 250-261. [CrossRef]

36. Wang, W.; Wu, Y.; Zhang, D. Association of dairy products consumption with risk of obesity in children and adults: A meta-analysis of mainly cross-sectional studies. Ann. Epidemiol. 2016, 26, 870-882. [CrossRef]

37. Patel, A.I.; Moghadam, S.D.; Freedman, M.; Hazari, A.; Fang, M.L.; Allen, I.E. The association of flavored milk consumption with milk and energy intake, and obesity: A systematic review. Prev. Med. (Baltim) 2018, 111, 151-162. [CrossRef] [PubMed]

38. Zheng, M.; Rangan, A.; Allman-Farinelli, M.; Rohde, J.F.; Olsen, N.J.; Heitmann, B.L. Replacing sugary drinks with milk is inversely associated with weight gain among young obesity-predisposed children. Br. J. Nutr. 2015, 114, 1448-1455. [CrossRef] [PubMed]

39. Zheng, M.; Rangan, A.; Olsen, N.J.; Andersen, L.B.; Wedderkopp, N.; Kristensen, P.; Grøntved, A.; Ried-Larsen, M.; Lempert, S.M.; Allman-Farinelli, M.; et al. Substituting sugar-sweetened beverages with water or milk is inversely associated with body fatness development from childhood to adolescence. Nutrition 2015, 31, 38-44. [CrossRef] [PubMed]

40. Keast, D.R.; Hill Gallant, K.M.; Albertson, A.M.; Gugger, C.K.; Holschuh, N.M. Associations between yogurt, dairy, calcium, and vitamin D intake and obesity among U.S. children aged 8-18 years: NHANES, 2005-2008. Nutrients 2015, 7, 1577-1593. [CrossRef] [PubMed]

41. Welsh, J.A.; Wang, Y.; Figueroa, J.; Brumme, C. Sugar intake by type (added vs. naturally occurring) and physical form (liquid vs. solid) and its varying association with children's body weight, NHANES 2009-2014. Pediatr. Obes. 2018, 13, 213-221. [CrossRef]

42. Marabujo, T.; Ramos, E.; Lopes, C. Dairy products and total calcium intake at 13 years of age and its association with obesity at 21 years of age. Eur. J. Clin. Nutr. 2018, 72, 541-547. [CrossRef] 
43. Fathi, Y.; Faghih, S.; Zibaeenezhad, M.J.; Tabatabaei, S.H.R. Kefir drink leads to a similar weight loss, compared with milk, in a dairy-rich non-energy-restricted diet in overweight or obese premenopausal women: A randomized controlled trial. Eur. J. Nutr. 2016, 55, 295-304. [CrossRef]

44. National Academies of Sciences Engineering and Medicine Dietary Reference Intakes for Sodium and Potassium. 2019. Available online: http://www.nationalacademies.org/hmd/Reports/2019/dietary-referenceintakes-sodium-potassium.aspx (accessed on 9 May 2019).

(c) (

(C) 2020 by the authors. Licensee MDPI, Basel, Switzerland. This article is an open access article distributed under the terms and conditions of the Creative Commons Attribution (CC BY) license (http://creativecommons.org/licenses/by/4.0/). 